\title{
RESEARCH
}

\section{Harmonic analysis and statistics of the first Galois cohomology group}

\author{
Brandon Alberts ${ }^{1 *}$ (D) and Evan O'Dorney ${ }^{2}$
}

${ }^{\text {*Correspondence: }}$

bralberts@ucsd.edu

${ }^{1}$ University of California San Diego, La Jolla, CA, USA

Full list of author information is available at the end of the article

\begin{abstract}
We utilize harmonic analytic tools to count the number of elements of the Galois cohomology group $f \in H^{1}(K, T)$ with discriminant-like invariant $\operatorname{inv}(f) \leq X$ as $X \rightarrow \infty$. Specifically, Poisson summation produces a canonical decomposition for the corresponding generating series as a sum of Euler products for a very general counting problem. This type of decomposition is exactly what is needed to compute asymptotic growth rates using a Tauberian theorem. These new techniques allow for the removal of certain obstructions to known results and answer some outstanding questions on the generalized version of Malle's conjecture for the first Galois cohomology group.
\end{abstract}

\section{Introduction}

Let $K$ be a number field. Extensions of $K$ with certain Galois groups and fixed resolvent can be parametrized by coclasses in the first cohomology group $H^{1}(K, T)$ of a Galois module $T$. It is natural to count such coclasses asymptotically by an invariant such as the norm of the discriminant or the product of the ramified primes.

The first author [1] proved a result counting coclasses by discriminant-like invariants which are Frobenian; that is, they are determined at almost all places $p$ of $K$ by the Frobenius element $\left(\frac{F / K}{p}\right)$ for some finite extension $F / K$. (We call the finitely many places where this is not satisfied irregular places.) Moreover, the result also applies if we impose a local condition $L_{p} \subseteq H^{1}\left(K_{p}, T\right)$ at each place $p$, provided that these conditions are again Frobenian in the appropriate sense. Explicitly, this result can be interpreted as giving the asymptotic size of an "infinite Selmer group," where for any family of local conditions $\mathcal{L}=\left(L_{p}\right)$ we define

$$
H_{\mathcal{L}}^{1}(K, T)=\left\{f \in H^{1}(K, T): \operatorname{res}_{p}(f) \in L_{p}\right\} .
$$

If we allow $L_{p}$ to include non-trivial ramification at infinitely many places, this set may be infinite. (For example, let $L_{p}=H^{1}\left(K_{p}, T\right)$ for all $p$ so that $H_{\mathcal{L}}^{1}(K, T)=H^{1}(K, T)$.) We can still say something about the "size" by talking about how the 1-coclasses are distributed with respect to some ordering. Given an admissible ordering inv : $H^{1}(K, T) \rightarrow I_{K}$ valued in the group of fractional ideals (as in [1]), we can define

$$
H_{\mathcal{L}, \text { inv }}^{1}(K, T ; X)=\left\{f \in H_{\mathcal{L}}^{1}(K, T): \mathcal{N}_{K / \mathbb{Q}}(\operatorname{inv}(f))<X\right\},
$$

(c) The Author(s) 2021. This article is licensed under a Creative Commons Attribution 4.0 International License, which permits use, sharing, adaptation, distribution and reproduction in any medium or format, as long as you give appropriate credit to the original author(s) and the source, provide a link to the Creative Commons licence, and indicate if changes were made. The images or other third party material in this article are included in the article's Creative Commons licence, unless indicated otherwise in a credit line to the material. If material is not included in the article's Creative Commons licence and your intended use is not permitted by statutory regulation or exceeds the permitted use, you will need to obtain permission directly from the copyright holder. To view a copy of this licence, visit http://creativecommons.org/licenses/by/4.0/. 
which is a finite set, and we can ask how this set grows as $X \rightarrow \infty$. We will often omit the "inv" from the subscript when it is clear from context.

We state the Asymptotic Wiles result proven in [1] for comparison:

Theorem (Asymptotic Wiles Theorem; Theorem 4.7 [1]) Let $T$ be a finite Galois $K$ module and $\mathcal{L}=\left(L_{p}\right)_{p}$ and inv a family of local conditions and admissible ordering, respectively. Suppose

(a) $\mathcal{L}$ and inv are Frobenian;

(b) $L_{p} \leq H^{1}\left(K_{p}, T\right)$ is a subgroup and, for almost all places $p$, $L_{p}$ contains $H_{u r}^{1}\left(K_{p}, T\right)$. For infinitely many places, $L_{p}$ is strictly larger than $H_{u r}^{1}\left(K_{p}, T\right)$;

(c) For all places $p$, if $f, f^{\prime} \in H^{1}\left(K_{p}, T\right)$ such that $\left\langle f||_{p}\right\rangle=\left\langle\left. f^{\prime}\right|_{I_{p}}\right\rangle \leq H^{1}\left(I_{p}, T\right)$, then $v_{p}(\operatorname{inv}(f))=v_{p}\left(\operatorname{inv}\left(f^{\prime}\right)\right)\left(\right.$ where $v_{p}(\mathfrak{a})$ equals the order to which $p$ divides $\left.\mathfrak{a}\right)$.

Then,

$$
\left|H_{\mathcal{L}}^{1}(K, T ; X)\right| \sim c_{\text {inv }}(K, \mathcal{L}) X^{1 / a_{\text {inv }}(\mathcal{L})}(\log X)^{b_{\text {inv }}(\mathcal{L})-1}
$$

for explicit positive integers $a_{\mathrm{inv}}(\mathcal{L})$ and $b_{\mathrm{inv}}(\mathcal{L})$, and an explicit positive real number $c_{\text {inv }}(K, \mathcal{L})$.

The method of proof in [1] consists of using the Greenberg-Wiles identity to rewrite the Dirichlet series counting such coclasses as a finite sum of Frobenian Euler products, and then applying a Tauberian theorem to extract information about the average size of the coefficients. Due to the close relationship to the Greenberg-Wiles identity, and viewing $\left|H_{\mathcal{L}}^{1}(K, T ; X)\right|$ as the asymptotic size of an "infinite Selmer group," this result is dubbed the Asymptotic Wiles theorem.

Given a compact box (i.e., some family of local conditions $\mathcal{L}=\left(L_{p}\right)$ for which $L_{p}$ is a subgroup of $H^{1}\left(K_{p}, T\right)$ at all places and $L_{p}=H_{u r}^{1}\left(K_{p}, T\right)$ at all but finitely many places), the Greenberg-Wiles identity acts as a local-to-global principle expressing $\left|H_{\mathcal{L}}^{1}(K, T)\right|$ as a product of local factors. Since Greenberg-Wiles requires the $L_{p}$ to be subgroups, some adjusting is necessary to get information about more general subsets such as $H_{\mathcal{L}}^{1}(K, T ; X)$. In particular, there is a need for hypothesis (c), which we might call the property of being constant on divisions, where a division of a group $G$ is a minimal subset $C \subset G$ closed under conjugation (i.e., $g \in C$ implies $g^{h} \in C$ ) and under invertible powers (i.e., if $n$ is coprime to the order of $G$ and $g \in C$, then $g^{n} \in C$ ). In particular, (c) implies that $v_{p}$ inv : $H^{1}\left(K_{p}, T\right) \rightarrow \mathbb{Z}$ is constant on divisions of the group $H^{1}\left(K_{p}, T\right)$ and is equivalent to requiring $v_{p}(\operatorname{inv}(f))$ to depend only on the subgroups of $H^{1}\left(I_{p}, T\right)$ in which $\left.f\right|_{I_{p}}$ lies for each $p$.

In this paper, we use harmonic analysis on adelic cohomology as a replacement for the Greenberg-Wiles identity, modeled after the celebrated use of harmonic analysis on the adeles in Tate's thesis [12]. Harmonic analysis on adelic cohomology has been used by Frei-Loughran-Newton $[5,6]$ as an alternate approach to counting abelian extensions of number fields with prescribed local conditions, and by the second author [10] to give a streamlined proof of the Ohno-Nakagawa reflection theorem and its generalizations. Poisson summation, in this setting, is a suitable generalization of Greenberg-Wiles that will allow us to lift some of the hypotheses in the main theorem to prove the following extended version of the Asymptotic Wiles Theorem: 
Theorem 1.1 Let $T$ be a finite Galois $K$-module and $\mathcal{L}=\left(L_{p}\right)_{p}$ and inv a family of local conditions and admissible ordering, respectively. Suppose

(a) $\mathcal{L}$ and inv are Frobenian.

(b) For all but finitely many places $p, L_{p}$ is a union of cosets of $H_{u r}^{1}\left(K_{p}, T\right)$ containing the identity coset.

Then,

$$
\left|H_{\mathcal{L}}^{1}(K, T ; X)\right| \sim c_{\text {inv }}(K, \mathcal{L}) X^{1 / a_{\text {inv }}(\mathcal{L})}(\log X)^{b_{\text {inv }}(\mathcal{L})-1}
$$

for explicit positive integers $a_{\mathrm{inv}}(\mathcal{L})$ and $b_{\mathrm{inv}}(\mathcal{L})$, and an explicit positive real number $c_{\text {inv }}(K, \mathcal{L})$.

This is a significant improvement, in particular weakening (b) by allowing $L_{p}$ to be non-subgroups and completely removing the constancy-on-divisions hypothesis (c) from the original result. Previously studied methods for counting number fields ordered by an arbitrary invariant (such as Wright's work on abelian extensions [13]) do not require a constancy-on-divisions assumption, suggesting that it is merely an artifact of the methods being used in [1]. By using harmonic analysis to bypass this condition, we verify this intuition.

As a particular example, if $G \subseteq S_{n}$ is a faithful transitive representation of $G$, the discriminant $\operatorname{disc}(\Sigma)$ of the degree- $n$ étale algebra attached to a coclass $\Sigma \in H^{1}(K, G)$ is a natural invariant to order coclasses in $H^{1}(K, G)$ by. (A more in-depth discussion of discriminants of étale algebras is found in [1, Definition 3.2].) It satisfies the constancy-on-divisions property (c) at all but finitely many places, but some issues occur at wildly ramified places. The first author uses this to prove upper and lower bounds on $\left|H_{\mathcal{L}}^{1}(K, T ; X)\right|$ of the same order of magnitude in [1] when $T \unlhd G$ has an induced Galois module structure given by $x . t=\pi(x) t \pi(x)^{-1}$ for some $\pi: G_{K} \rightarrow G$ and remarks that (c) is the obstruction to producing the asymptotic main term. (In this setting, $H^{1}(K, T) \rightarrow H^{1}(K, G)$ has finite kernel, and thus, $H^{1}(K, T)$ can still be ordered by the discriminant of the étale algebra corresponding to the image in $H^{1}(K, G)$.) Theorem 1.1 is a sufficient generalization to prove the following corollary, which counts non-abelian extensions with specified non-abelian Galois group $G$ and specified resolvent by an abelian normal subgroup. In the language of [1], this counts ( $T \unlhd G$ )-towers ordered by discriminant, where $T$ is an abelian normal subgroup of $G$.

Corollary 1.2 Let $T$ be an abelian normal subgroup of a finite group $G$ and $\pi$ : $\mathrm{Gal}(\bar{K} / K) \rightarrow G$ a homomorphism with $T \pi(\mathrm{Gal}(\bar{K} / K))=G$ (or equivalently $\pi$ surjects onto $G / T)$, defining a Galois action on $T$ by x.t $=\pi(x) t \pi(x)^{-1}$.

(i) If $\mathcal{L}$ and inv satisfy the hypotheses of Theorem 1.1 and $S$ is the set of irregular places, then the limit

$$
\lim _{X \rightarrow \infty} \frac{\mid\left\{f \in H_{\mathcal{L}}^{1}(K, T ; X): f * \pi \text { surjective }\right\} \mid}{\left|H_{\mathcal{L}}^{1}(K, T ; X)\right|}
$$

converges, where $(f * \pi)(x)=f(x) \pi(x)$ is understood to apply to a representative off in $Z^{1}(K, T)$. Moreover, the limit is 
(a) positive if $\pi$ is surjective,

(b) positive if $T=\left\langle f_{p}\left(I_{p}\right): f_{p} \in L_{p}, p \notin S\right\rangle$, and

(c) equal to 1 if $T=\left\langle f_{p}\left(I_{p}\right): f_{p} \in L_{p}^{\left[a_{\text {inv }}(\mathcal{L})\right]}, p \notin S\right\rangle$ where $L_{p}^{[m]}=\left\{f \in L_{p}\right.$ : $\left.v_{p}(\operatorname{inv}(f))=m\right\}$.

(ii) If $G \subset S_{n}$ is a transitive representation of $G$, then the invariant

$$
\operatorname{disc}_{\pi}(f)=\operatorname{disc}(f * \pi)
$$

satisfies the hypotheses of Theorem 1.1, where disc : $\operatorname{Hom}\left(\operatorname{Gal}(\bar{K} / K), S_{n}\right) \rightarrow I_{K}$ is the discriminant on the associated étale algebra of degree $n$.

Corollary 1.2 is a generalization of [1, Theorem 5.3], and for the most part, the proof is the same after replacing the Asymptotic Wiles theorem in [1] with the more powerful theorem 1.1. In particular, as described in [1], $\operatorname{disc}_{\pi}$ satisfies condition $(i)(b)$ so that the number of towers with bounded discriminant

$$
N(L / K, T \unlhd G ; X)=\mid\left\{f \in H_{\operatorname{disc}_{\pi}}^{1}(K, T ; X): f * \pi \text { surjective }\right\} \mid
$$

is asymptotic to

$$
c X^{1 / a(T)}(\log X)^{b(K, T(\pi))-1}
$$

for some positive constant $c$ as $X \rightarrow \infty$. This proves the generalization of Malle's conjecture to towers for $T$ abelian described in [1] with an exact asymptotic.

In addition to allowing us to relax the hypotheses and prove the asymptotic main terms, the harmonic analytic method has noteworthy elegance. The proof found in this note is many times shorter than the original proof in [1], and it naturally motivates the resulting finite sum of Euler products as the sum over the dual arising in a Poisson summation.

\section{Fourier analysis on adelic cohomology}

Our goal is to study the average value of certain functions over the global cohomology classes $H^{1}(K, T)$. Given such a function $w$, we are looking to understand the summation

$$
\sum_{f \in H^{1}(K, T)} w(f)
$$

This can appear as an average of an arithmetic function if $w(f)=w_{X}(f)$ is zero when $\operatorname{inv}(f) \geq X$, so that we can consider the limiting behavior as $X \rightarrow \infty$. It can also appear as a Dirichlet series, such as when $w(f)=\operatorname{inv}(f)^{-s}$, of which we are interested in studying the meromorphic continuation. (Note that in many cases, these two examples are intimately related, and results for one can imply results for the other.)

The idea is to realize $H^{1}(K, T)$ as a discrete subgroup of some locally compact group, so that $\sum w(f)$ appears to be one side of a Poisson summation. We recall the Poisson formula for locally compact abelian groups (see, for instance, Hewitt and Ross [7], p. 246):

Theorem 2.1 Let $G$ be a locally compact abelian group and $w: G \rightarrow \mathbb{C}$ a continuous function of class $L^{1}$. Suppose that $G_{0} \subseteq G$ is a closed subgroup such that

(a) For each $x \in G$, the integral

$$
w^{\prime}(x)=\int_{y \in G_{0}} w(x+y) \mathrm{d} y
$$

is absolutely convergent; 
(b) The resulting function $w^{\prime}: G / G_{0} \rightarrow \mathbb{C}$ is $L^{1}$;

(c) Its Fourier transform $\widehat{w^{\prime}}$ is $L^{1}$ on $\left(G / G_{0}\right)^{\vee} \cong G^{\vee} / G_{0}^{\perp}$ (where $G_{0}^{\perp}$ is the annihilator of $\left.G_{0}\right)$.

Then, with respect to an appropriate scaling of the measures on $G_{0}$ and $G_{0}^{\perp}$,

$$
\int_{x \in G_{0}} w(x) \mathrm{d} x=\int_{y \in G_{0}^{\perp}} \hat{w}(y) \mathrm{d} y .
$$

In our case, we will realize $G_{0}=H^{1}(K, T)$ as a discrete group, so that

$$
\int_{f \in H^{1}(K, T)} w(f) \mathrm{d} f=\sum_{f \in H^{1}(K, T)} w(f)
$$

(up to scaling the measure).

The goal of this section is to set up the other features of Poisson summation in this setting, including the definition of $G$ and the corresponding groups $G^{\vee}$ and $G_{0}^{\perp}$.

Definition 2.2 The adelic cohomology of a Galois $K$-module $T$ is the restricted direct product

$$
H^{1}\left(\mathbb{A}_{K}, T\right):=\prod_{p}^{\prime} H^{1}\left(K_{p}, T\right)_{H_{u r}^{1}\left(K_{p}, T\right)},
$$

or more explicitly

$$
H^{1}\left(\mathbb{A}_{K}, T\right):=\left\{\left(f_{p}\right) \in \prod_{p} H^{1}\left(K_{p}, T\right) \mid f_{p} \in H_{u r}^{1}\left(K_{p}, T\right) \text { for all but finitely many } p\right\} .
$$

When each $H^{1}\left(K_{p}, T\right)$ is given the discrete topology, $H^{1}\left(\mathbb{A}_{K}, T\right)$ is a locally compact abelian group. Its Pontryagin dual, by local Tate duality, is isomorphic to $H^{1}\left(\mathbb{A}_{K}, T^{*}\right)$ where $T^{*}=\operatorname{Hom}(T, \mu)$ is the Tate dual of $T$.

There exists a map

$$
i_{T}: H^{1}(K, T) \rightarrow H^{1}\left(\mathbb{A}_{K}, T\right)
$$

coming from a choice of embeddings $\bar{K} \hookrightarrow \bar{K}_{p}$, which we fix throughout. We gather the necessary information on this map from the Poitou-Tate nine-term exact sequence $[9$, Theorem 8.6.10], which in the case that $S$ is the set of all places is given by

$$
\begin{gathered}
0 \longrightarrow H^{0}(K, T) \longrightarrow H^{0}\left(\mathbb{A}_{K}, T\right) \longrightarrow H^{2}\left(K, T^{*}\right)^{\vee} \longrightarrow \\
\longrightarrow H^{1}(K, T) \stackrel{i_{T}}{\longrightarrow} H^{1}\left(\mathbb{A}_{K}, T\right) \stackrel{\left(i_{T^{*}}\right)^{\vee}}{\longrightarrow} H^{1}\left(K, T^{*}\right)^{\vee} \longrightarrow \\
\longrightarrow H^{2}(K, T) \longrightarrow H^{2}\left(\mathbb{A}_{K}, T\right) \longrightarrow H^{0}\left(K, T^{*}\right)^{\vee} \longrightarrow 0
\end{gathered}
$$

where each of the homomorphisms are continuous and the nine terms have the following topologies, respectively: finite, compact, compact, discrete, locally compact, compact, discrete, discrete, finite.

Although $i_{T}$ need not be injective, we see that the kernel is the continuous image of a compact group into a discrete cocompact group, and so is necessarily finite. Moreover, the middle row shows that the images of $i_{T}$ and $i_{T^{*}}$ are dual lattices in their respective cohomology groups. We thus derive the following corollary of Poisson summation: 
Theorem 2.3 Normalize the measure on $H^{1}\left(K_{p}, T\right)$ so that each single point has measure

$$
\frac{1}{\left|H^{0}\left(K_{p}, T\right)\right|},
$$

and take the product of these measures as a measure on $H^{1}\left(\mathbb{A}_{K}, T\right)$ so that the compact subgroup

$$
\prod_{p \text { finite }} H_{u r}^{1}\left(K_{p}, T\right)
$$

has measure

$$
\prod_{p \mid \infty} \frac{1}{\left|H^{0}\left(K_{p}, M\right)\right|} .
$$

Then, for any function $w: H^{1}\left(\mathbb{A}_{K}, T\right) \rightarrow \mathbb{C}$ satisfying the hypotheses of Theorem 2.1 for $G=H^{1}\left(\mathbb{A}_{K}, T\right)$ and $G_{0}=H^{1}(K, T)$,

$$
\sum_{f \in H^{1}(K, T)} w(f)=\frac{\left|H^{0}(K, T)\right|}{\left|H^{0}\left(K, T^{*}\right)\right|} \sum_{g \in H^{1}\left(K, T^{*}\right)} \hat{w}(g),
$$

where we abuse notation and write $w: H^{1}(K, T) \rightarrow \mathbb{C}$ for the pullback of $w: H^{1}\left(\mathbb{A}_{K}, T\right) \rightarrow$ $\mathbb{C}$ along $i_{T}$.

Proof The image $\operatorname{im}\left(\iota_{T}\right)$ is a discrete subgroup of $H^{1}\left(\mathbb{A}_{K}, T\right)$, and the Poitou-Tate nineterm exact sequence implies $\operatorname{im}\left(\iota_{T^{*}}\right)=\operatorname{im}\left(\iota_{T}\right)^{\perp}$. Thus, Poisson summation (Theorem 2.1) implies that there exists a constant $c$ such that

$$
\int_{f \in \operatorname{im}\left(\iota_{T}\right)} w(f) \mathrm{d} f=c \cdot \int_{g \in \operatorname{im}\left(\iota_{T} *\right)} \hat{w}(g) \mathrm{d} g .
$$

The constant $c$ depends on how the measures $\mathrm{d} f$ and $\mathrm{d} g$ are scaled. We specified a scaling at the beginning, so we cannot assume the two integrals are equal on the nose. We know that $\operatorname{im}\left(\iota_{T}\right)$ and $\operatorname{im}\left(\iota_{T^{*}}\right)$ are discrete, so that the above is equivalent to

$$
\sum_{f \in \operatorname{im}\left(\iota_{T}\right)} w(f)=c \cdot \sum_{g \in \operatorname{im}\left(\iota_{T^{*}}\right)} \hat{w}(g) .
$$

Recalling that $w$ is defined on $H^{1}\left(\mathbb{A}_{K}, T\right)$ and that the corresponding map $w: H^{1}(K, T) \rightarrow$ $\mathbb{C}$ is defined to be the pullback along $\iota_{T}$, we see in particular that $w(f h)=w(f)$ for any $h \in \operatorname{ker}\left(\iota_{T}\right)$. That same statement is true for $T$ replaced by $T^{*}$, so

$$
\sum_{f \in H^{1}(K, T)} w(f)=\frac{c\left|\operatorname{ker}\left(\iota_{T^{*}}\right)\right|}{\left|\operatorname{ker}\left(\iota_{T}\right)\right|} \sum_{g \in H^{1}\left(K, T^{*}\right)} \hat{w}(g) .
$$

It now suffices to determine the constant coefficient

$$
c^{\prime}=\frac{c\left|\operatorname{ker}\left(\iota_{T^{*}}\right)\right|}{\left|\operatorname{ker}\left(\iota_{T}\right)\right|},
$$

which is independent of $w$. For this, we can simply take for $w$ any function for which either sum in (1) is nonzero.

We note that when $w=\mathbf{1}_{L}$ is the characteristic function of a compact open box $L=$ $\prod_{p} L_{p}$, the left-hand side of (1) is the cardinality of the Selmer group

$$
\operatorname{Sel}(L)=\left\{f \in H^{1}(K, T): f \in L_{p} \forall p\right\} .
$$


(If $i_{T}$ has non-trivial kernel, it is necessarily contained in $\operatorname{Sel}(L)$ for every $L$.) With our normalizations, the Fourier transform of $w_{p}=\mathbf{1}_{L_{p}}$ as a weighting on $H^{1}\left(K_{p}, T\right)$ is

$$
\hat{w}_{p}=\frac{\left|L_{p}\right|}{\left|H^{0}(K, T)\right|} \mathbf{1}_{L_{\vec{p}}^{\perp}}
$$

and by the multiplicative definition of the pairing,

$$
\hat{w}=\prod_{p} \hat{w}_{p}=\prod_{p} \frac{\left|L_{p}\right|}{\left|H^{0}(K, T)\right|} \mathbf{1}_{L^{\perp}} .
$$

So (1) becomes an identity relating the Selmer groups of $L$ and $L^{\perp}$ :

$$
\frac{|\operatorname{Sel}(L)|}{\left|\operatorname{Sel}\left(L^{\perp}\right)\right|}=c^{\prime} \prod_{p} \frac{\left|L_{p}\right|}{\left|H^{0}\left(K_{p}, T\right)\right|} .
$$

This identity is none other than the Greenberg-Wiles formula

$$
\frac{|\operatorname{Sel}(L)|}{\left|\operatorname{Sel}\left(L^{\perp}\right)\right|}=\frac{\left|H^{0}(K, T)\right|}{\left|H^{0}\left(K, T^{*}\right)\right|} \prod_{p} \frac{\left|L_{p}\right|}{\left|H^{0}\left(K_{p}, T\right)\right|}
$$

(see [4, Theorem 2.19] or [8, Theorem 3.11]), where the constant coefficient

$$
c^{\prime}=\frac{\left|H^{0}(K, T)\right|}{\left|H^{0}\left(K, T^{*}\right)\right|}
$$

arises out of a global Euler characteristic computation.

The use of Theorem 2.3 separates into two cases:

1. If $w$ is periodic with respect to a compact open subgroup of the form

$$
\prod_{p \notin S} H_{u r}^{1}\left(K_{p}, T\right)
$$

for some finite set of places $S$, then $\hat{w}(g)$ has finite support. This implies that the sum over $H^{1}\left(K, T^{*}\right)$ is secretly a finite sum. In this case, it will suffice to understand the behavior of $\hat{w}(g)$ at individual values of $g$. (We note that this is similar to classical Poisson summation for real-valued functions, where the Fourier transform of a periodic function has discrete support. The extra structure of being periodic with respect to an open subgroup is what gives an even smaller support in this case.)

2. If $w$ is not periodic, then the sum over $H^{1}\left(K, T^{*}\right)$ is truly an infinite sum. This situation is worth considering, as we can show that it is closely related to other important questions in number theory and arithmetic statistics. While dealing with the infinite sum requires more powerful analytic tools than we will employ in this paper, the behavior of each individual summand $\hat{w}(g)$ will give important insight into more general cases of restricting local conditions.

We will study examples of both periodic and non-periodic $w$ functions which are important to open questions in number theory, and in the periodic case, we will use these results to prove Theorem 1.1.

\section{Multiplicative $w$}

The examples we will consider come from multiplicative functions: 
Definition 3.1 We call a function $w: H^{1}\left(\mathbb{A}_{K}, T\right) \rightarrow \mathbb{C}$ multiplicative if

$$
w(f)=\prod_{p} w\left(\left.f\right|_{G_{K_{p}}}\right) .
$$

The discriminant is multiplicative, so the function $w(f)=\operatorname{disc}(f)^{-s}$ is necessarily multiplicative (as is the corresponding example for any admissible ordering inv as defined in [1, Definition 4.4]). Multiplicativity is preserved by the Fourier transform, so that the identification of $H^{1}\left(K_{p}, T\right)^{\vee}$ with $H^{1}\left(K_{p}, T^{*}\right)$ by Tate duality implies

$$
\begin{aligned}
\hat{w}(g) & =\prod_{p} \hat{w}\left(\left.g\right|_{G_{K_{p}}}\right) \\
& =\prod_{p}\left(\frac{1}{\left|H^{0}\left(K_{p}, T\right)\right|} \sum_{f_{p} \in H^{1}\left(K_{p}, T\right)}\left\langle f_{p},\left.g\right|_{G_{K_{p}}}\right\rangle w\left(f_{p}\right)\right)
\end{aligned}
$$

where $\langle\cdot, \cdot\rangle$ is the Tate pairing and we recall that each point in $H^{1}\left(K_{p}, T\right)$ was assigned measure $1 /\left|H^{0}\left(K_{p}, T\right)\right|$. Note that if $g=0$ is the identity, this is reminiscent of the local series given by Bhargava in the Malle-Bhargava principle [1,2]. Indeed, if $w(f)=\operatorname{disc}(f)^{-s}$, then it follows that

$$
\hat{w}(0)=\prod_{p}\left(\frac{1}{\left|H^{0}\left(K_{p}, T\right)\right|} \sum_{f_{p} \in H^{1}\left(K_{p}, T\right)} \operatorname{disc}\left(f_{p}\right)^{-s}\right)
$$

is exactly the local series given by Bhargava (in the case of the trivial action) and the first author (in the case of non-trivial actions). This suggests a reformulation of the MalleBhargava principle in terms of Fourier transforms:

Heuristic 3.2 (Malle-Bhargava principle on $H^{1}(K, T)$ ) Suppose $w: H^{1}\left(\mathbb{A}_{K}, T\right) \rightarrow \mathbb{C}$ is a "reasonable" multiplicative function satisfying the hypotheses of Poisson summation (Theorem 2.1). Then, there exists a positive constant $c>0$ such that

$$
\sum_{f \in H^{1}(K, T)} w(f)=c \cdot \hat{w}(0)+\text { "lower-order term." }
$$

Following in the footsteps of Cohen-Lenstra [3], we do not give a definition for a "reasonable" function; instead, we will consider a series of examples in this paper that should be considered "reasonable." The notion of a "lower-order term" depends on the context as well, and we do not give an explicit definition in all cases. In the example $w(f)=\operatorname{disc}(f)^{-s}, \hat{w}(0)$ is a Dirichlet series whose rightmost pole is at $s=1 / a(T)$ of order $b(K, T)$ [1, Theorem 3.3]. We then take "lower-order term" to mean a Dirichlet series whose rightmost pole is at $s \in\{z \in \mathbb{C}: \operatorname{Re}(z)<1 / a(T)\} \cup\{1 / a(T)\}$, and if it it $s=1 / a(T)$, then it is of an order $<b(K, T)$. By applying a Tauberian theorem, we see that this expression identifies the sum of coefficients of $\hat{w}(0)$ as the main term of

$$
\sum_{\operatorname{disc}(f)<X} 1,
$$

while the "lower-order term" converts into an error of a smaller magnitude.

Remark In the discriminant ordering (or other similar orderings), there are certain cases where the main term really is a constant multiple of $\hat{w}(0)$. This will happen when there 
exist other $g \in H^{1}\left(K, T^{*}\right)$ where $\hat{w}(g)$ is on the same "order of magnitude" as $\hat{w}(0)$ and so must also contribute to the main term.

One easily shows that $|\hat{w}(g)| \leq|\hat{w}(0)|$ for all $g \in H^{1}\left(K, T^{*}\right)$ and all $w$ for which the Fourier transform exists, which suggests that $\hat{w}(0)$ should attain the correct order of magnitude, if not the correct multiplicity. (Note: there is certainly something to prove here, as we have done nothing to show that the infinite sum of $\hat{w}(g)$ does not attain a larger order of magnitude than the individual summands. This is why we merely refer to this statement as a "heuristic.")

\section{Periodic $w$}

Suppose $w$ is periodic with respect to a compact open subgroup $Y \leq H^{1}\left(\mathbb{A}_{K}, T\right)$ and satisfies the hypotheses of Theorem 2.3. Then, $\hat{w}$ is supported on the compact open subgroup $Y^{\perp}$, and

$$
\sum_{g \in H^{1}\left(K, T^{*}\right)} \hat{w}(g)=\sum_{g \in H^{1}\left(K, T^{*}\right) \cap Y^{\perp}} \hat{w}(g)
$$

is a sum over the group $H^{1}\left(K, T^{*}\right) \cap Y^{\perp}$. Discreteness of $H^{1}\left(K, T^{*}\right)$ and compactness of $Y^{\perp}$ imply that $H^{1}\left(K, T^{*}\right) \cap Y^{\perp}$ is finite, which makes the summation easier to evaluate.

An important example of a periodic function is the absolute discriminant when $T$ has the trivial action. If

$$
Y=\prod_{p \nmid \infty} \operatorname{Hom}_{u r}\left(\mathbb{Q}_{p}, T\right),
$$

then $|\operatorname{disc}(f+y)|=|\operatorname{disc}(f)|$ for every $y \in Y$, as the discriminant only sees ramification behavior. $Y \leq H^{1}\left(\mathbb{A}_{\mathbb{Q}}, T\right)$ is necessarily a compact open subgroup in the adelic topology. The function $w(f)=|\operatorname{disc}(f)|^{-s}$ is shown to satisfy the hypotheses of Theorem 2.3 by [5], which they then use to compute the location and orders of poles of the corresponding Dirichlet series.

Our main example of a periodic function is a generalization of the absolute discriminant to the largest class of functions for which the same ideas will work. This follows the ideas in [1] by allowing non-trivial actions on $T$, allowing restricted local conditions $\mathcal{L}=\left(L_{p}\right)$, and allowing an admissible ordering to be used instead of the usual discriminant:

Proposition 4.1 Let $K$ be a number field, $S$ a finite set of places of $K$, and $T$ a Galois module. If

(a) $\mathcal{L}=\left(L_{p}\right)$ is a family of subsets $L_{p} \subset H^{1}\left(K_{p}, T\right)$ such that for all $p \notin S, L_{p}$ is closed under translation by $H_{u r}^{1}\left(K_{p}, T\right)$ and $0 \in L_{p}$;

(b) inv is an admissible ordering as defined in [1, Definition 4.4],

then the function $w: H^{1}\left(\mathbb{A}_{K}, T\right) \rightarrow \mathbb{C}$ defined by

$$
w(f)= \begin{cases}\mathcal{N}_{K / \mathbb{Q}}(\operatorname{inv}(f))^{-s} & f \in \prod_{p} L_{p} \\ 0 & f \notin \prod_{p} L_{p}\end{cases}
$$

satisfies the hypotheses of Theorem 2.3 and is periodic with respect to the compact open subgroup

$$
Y=Y_{S}=\prod_{p \notin S} H_{u r}^{1}\left(K_{p}, T\right) .
$$


Proof $w$ is periodic with respect to $Y$ by definition, so it suffices to check the hypotheses of Poisson summation.

We first prove that $w$ is $L^{1}$ on $H^{1}\left(\mathbb{A}_{K}, T\right)$ for $\Re(s)$ sufficiently large. Define

$$
a(w)=\liminf _{\mathcal{N}_{K / \mathbb{Q}} p \rightarrow \infty} \min _{\substack{f \in H^{1}\left(K_{p}, T\right) \\ v_{p}(\operatorname{inv}(f)) \neq 0}} v_{p}(\operatorname{inv}(f)) .
$$

Then,

$$
\int_{H^{1}\left(\mathbb{A}_{K}, T\right)}|w(f)| \mathrm{d} f \ll \prod_{p}\left(1+O\left(\mathcal{N}_{K / \mathbb{Q}}(p)^{-a(w) \Re(s)}\right)\right),
$$

which converges absolutely for $\Re(s)>1 / a(w)$. We use this to prove each of the hypotheses of Poisson summation (Theorem 2.1) with $G=H^{1}\left(\mathbb{A}_{K}, T\right)$ and $G_{0}=\operatorname{im}\left(\iota_{T}\right)$ :

(a) Since $w$ is $Y$-periodic, we have

$$
|w(x)|=\frac{1}{\mu(Y)} \int_{Y}|w(x+y)| \mathrm{d} y,
$$

where since $Y$ is open, the Haar measures on $Y$ and $G$ can be taken to coincide. Hence,

$$
\begin{aligned}
\int_{G_{0}}|w(x+z)| \mathrm{d} z & =\frac{1}{\mu(Y)} \int_{G_{0}} \int_{Y}|w(x+y+z)| \mathrm{d} y \mathrm{~d} z \\
& =\frac{\left|G_{0} \cap Y\right|}{\mu(Y)} \int_{Y+G_{0}}|w(x+z)| \mathrm{d} z \\
& \leq \frac{\left|G_{0} \cap Y\right|}{\mu(Y)} \int_{G}|w(z)| \mathrm{d} z<\infty
\end{aligned}
$$

where we used that since $Y$ is compact and $G_{0}$ is discrete, their intersection is finite. Thus, the integral defining $w^{\prime}$ is absolutely convergent.

(b) We note that

$$
\begin{aligned}
\int_{G / G_{0}}\left|w^{\prime}(x)\right| \mathrm{d} x & =\int_{G / G_{0}}\left|\int_{G_{0}} w(x+y) \mathrm{d} y\right| \mathrm{d} x \\
& \leq \int_{G / G_{0}} \int_{G_{0}}|w(x+y)| \mathrm{d} y \mathrm{~d} x \\
& =\int_{G}|w(x)| \mathrm{d} x,
\end{aligned}
$$

which converges because $w$ is $L^{1}$ for $\Re(s)>1 / a(w)$, and thus, $w^{\prime}$ is $L^{1}$ for $\Re(s)>$ $1 / a(w)$.

(c) Since $w^{\prime}$ is $L^{1}$, its Fourier transform $\widehat{w^{\prime}}$ is bounded and hence $L^{1}$ on the compact space $G^{\vee} / G_{0}^{\perp}$.

This is almost precisely the setup for Theorem 1.1, where the only condition missing is that $\mathcal{L}$ and inv are Frobenian. Indeed, Proposition 4.1 implies that

$$
\sum_{f \in H^{1}(K, T)} w(f)
$$


is a Dirichlet series which can be expressed as a finite sum of Euler products. Knowing that $\mathcal{L}$ and inv are Frobenian implies that the Euler products are of Frobenian factors, which is sufficient for calculating the locations of their poles as is done in [1, Proposition 2.2]. A Tauberian theorem is then used to convert the analytic information at the poles into asymptotic information. We sketch the argument here, but refer to [1] for the details.

Proof of Theorem 1.1 Let $w$ be as in Proposition 4.1. Then,

$$
\sum_{f \in H^{1}(K, T)} w(f)=\frac{\left|H^{0}(K, T)\right|}{\left|H^{0}\left(K, T^{*}\right)\right|} \sum_{g \in H^{1}\left(K, T^{*}\right)} \hat{w}(g)
$$

is a Dirichlet series. $w$ is periodic with respect to $Y$, which implies $\hat{w}$ has finite support in $H^{1}\left(K, T^{*}\right)$. The rate of growth of $\left|H_{\mathcal{L}}^{1}(K, T ; X)\right|$ is determined by the location and order of the rightmost pole of this series via a Tauberian theorem. It then suffices to show that the rightmost pole occurs at $1 / a_{\text {inv }}(\mathcal{L})$ of order $b_{\text {inv }}(\mathcal{L})$.

First, consider

$$
\hat{w}(0)=\prod_{p}\left(\frac{1}{\left|H^{0}\left(K_{p}, T\right)\right|} \sum_{f \in L_{p}} \mathcal{N}_{K / \mathbb{Q}}(\operatorname{inv}(f))^{-s}\right) .
$$

This is precisely the Frobenian Euler product studied in [1], denoted $Q(0, s)$ in Propositions 4.14 and 4.16 of that paper, which was shown to have rightmost pole at $1 / a_{\text {inv }}(\mathcal{L})$ of order $b_{\text {inv }}(\mathcal{L})$. For each other term, note that

$$
\begin{aligned}
|\hat{w}(g)| & =\left|\prod_{p}\left(\frac{1}{\left|H^{0}\left(K_{p}, T\right)\right|} \sum_{f \in L_{p}}\left\langle f,\left.g\right|_{G_{K_{p}}}\right\rangle \mathcal{N}_{K / \mathbb{Q}}(\operatorname{inv}(f))^{-s}\right)\right| \\
& \leq \prod_{p}\left(\frac{1}{\left|H^{0}\left(K_{p}, T\right)\right|} \sum_{f \in L_{p}} \mathcal{N}_{K / \mathbb{Q}}(\operatorname{inv}(f))^{-\Re(s)}\right),
\end{aligned}
$$

which agrees with $\hat{w}(0)$ at $\Re(s)$. Taking a limit as $s \rightarrow 1 / a_{\text {inv }}(\mathcal{L})$ implies that if $\hat{w}(g)$ has a pole (or a "fractional pole," i.e., a branch singularity like $\left(s-1 / a_{\text {inv }}(\mathcal{L})\right)^{-5 / 2}$, for example) at $s=1 / a_{\text {inv }}(\mathcal{L})$, it is of order at most $b_{\text {inv }}(\mathcal{L})$. Thus, after adding the finitely many other terms $\hat{w}(g)$ to $\hat{w}(0)$, it suffices to show that this rightmost pole of $\hat{w}(0)$ does not cancel out.

The proof that the poles do not cancel out is the same as the proof of the Asymptotic Wiles theorem in [1], so we only summarize the main points. Write $L_{p}^{[m]}=\left\{f \in L_{p}\right.$ : $\left.v_{p}(\operatorname{inv}(f))=m\right\}$. Let $T^{\prime} \leq T$ be the subgroup defined by

$$
T^{\prime}=\left\langle f_{p}\left(I_{p}\right): p \notin S, f_{p} \in L_{p}^{\left[a_{\mathrm{inv}}(\mathcal{L})\right]}\right\rangle,
$$

and let $i: T^{\prime} \rightarrow T$ denote the inclusion. The restrictions $L\left(T^{\prime}\right)_{p}=\left(i_{*}\right)^{-1}\left(L_{p}\right) \subseteq$ $H^{1}\left(K_{p}, T^{\prime}\right)$ form an admissible family $\mathcal{L}\left(T^{\prime}\right)$ of local conditions on $H^{1}\left(K, T^{\prime}\right)$, and $i_{*}: H_{\mathcal{L}\left(T^{\prime}\right)}^{1}\left(K, T^{\prime} ; X\right) \rightarrow H_{\mathcal{L}}^{1}(K, T ; X)$ has finite kernel. Consider $p \nmid|G| \infty$ such that $I_{p}$ acts trivially on $T$ and $p$ is a non-exceptional place so that $H_{u r}^{1}(K, T) \subseteq L_{p}$ and $L_{p}$ is closed under translation by $H_{u r}^{1}\left(K_{p}, T\right)$. The equality $\left|H^{0}\left(K_{p}, T\right)\right|=\left|H_{u r}^{1}\left(K_{p}, T\right)\right|$ implies

$$
\frac{\left|L_{p}^{\left[a_{\text {inv }}(\mathcal{L})\right]}\right|}{\left|H^{0}\left(K_{p}, T\right)\right|}=\frac{\left|L_{p}^{\left[a_{\text {inv }}(\mathcal{L})\right]}\right|}{\left|H_{u r}^{1}\left(K_{p}, T\right)\right|} .
$$


The first three terms of the inflation-restriction sequence

$$
0 \longrightarrow H_{u r}^{1}\left(K_{p}, T\right) \longrightarrow H^{1}\left(K_{p}, T\right) \stackrel{\operatorname{res}_{I_{p}}}{\longrightarrow} H^{1}\left(I_{p}, T\right) \longrightarrow H_{u r}^{2}(K, T)
$$

imply that $\left|L_{p}\right| /\left|H_{u r}^{1}\left(K_{p}, T\right)\right|=\left|\operatorname{res}_{I_{p}}\left(L_{p}\right)\right|$. The fact that $v_{p} \operatorname{inv}(f)$ depends only on $\left.f\right|_{I_{p}}$ implies that $L_{p}^{\left[a_{\text {inv }}(\mathcal{L})\right]} \subset L_{p}$ is also a union of cosets of $H_{u r}^{1}\left(K_{p}, T\right)$, which implies

$$
\frac{\left|L_{p}^{\left[a_{\text {inv }}(\mathcal{L})\right]}\right|}{\left|H_{u r}^{1}\left(K_{p}, T\right)\right|}=\left|\operatorname{res}_{I_{p}}\left(L_{p}^{\left[a_{\text {inv }}(\mathcal{L})\right]}\right)\right| .
$$

We now claim that $i_{*} L\left(T^{\prime}\right)_{p}^{\left[a_{\text {inv }}(\mathcal{L})\right]} H_{u r}^{1}\left(K_{p}, T\right)=L_{p}^{\left[a_{\text {inv }}(\mathcal{L})\right]}$, so that in particular $\left|\operatorname{res}_{I_{p}}\left(L_{p}^{\left[a_{\text {inv }}(\mathcal{L})\right]}\right)\right|=$ $\left|\operatorname{res}_{I_{p}}\left(i_{*} L\left(T^{\prime}\right)_{p}^{\left[a_{\text {inv }}(\mathcal{L})\right]}\right)\right|$. The containment

$$
i_{*} L\left(T^{\prime}\right)_{p}^{\left[a_{\text {inv }}(\mathcal{L})\right]} H_{u r}^{1}\left(K_{p}, T\right) \subseteq L_{p}^{\left[a_{\text {inv }}(\mathcal{L})\right]}
$$

is clear by construction. To prove the other containment, suppose $f \in L_{p}^{\left[a_{\text {inv }}(\mathcal{L})\right]}$. The fact that $I_{p}$ acts trivially on $T$ implies that $\operatorname{res}_{I_{p}}(f) \in \operatorname{Hom}\left(I_{p}, T\right)$. The coclass $f$ also satisfies $v_{p}(\operatorname{inv}(f))=a_{\text {inv }}(\mathcal{L})$ by definition of $L_{p}^{\left[a_{\text {inv }}(\mathcal{L})\right]}$, which by construction implies $f\left(I_{p}\right) \leq T^{\prime}$ so that really $\operatorname{res}_{I_{p}}(f) \in \operatorname{Hom}\left(I_{p}, T^{\prime}\right) \subseteq \operatorname{Hom}\left(I_{p}, T\right)$. Surjectivity of $\operatorname{res}_{I_{p}}$ (which follows, for instance, from $H_{u r}^{2}\left(K_{p}, T\right)=0$ in the inflation-restriction sequence [11, section 5, Proposition 18(c)]) implies that there exists some $f^{\prime} \in H^{1}\left(K_{p}, T^{\prime}\right)$ with $\operatorname{res}_{I_{p}}\left(f^{\prime}\right)=\operatorname{res}_{I_{p}}(f)$. In particular, $v_{p}\left(\operatorname{inv}\left(f^{\prime}\right)\right)=v_{p}(\operatorname{inv}(f))$ as this is determined entirely by the restriction to $I_{p}$, so $f^{\prime} \in L\left(T^{\prime}\right)_{p}^{\left[a_{\text {inv }}(\mathcal{L})\right]}$ and $f \in i_{*}\left(f^{\prime}\right) \operatorname{ker}\left(\operatorname{res}_{I_{p}}\right) \subseteq i_{*} L\left(T^{\prime}\right)_{p}^{\left[a_{\text {inv }}(\mathcal{L})\right]} H_{u r}^{1}\left(K_{p}, T\right)$.

Noting that $i_{*}: H^{1}\left(I_{p}, T^{\prime}\right) \rightarrow H^{1}\left(K_{p}, T\right)$ is injective when $I_{p}$ acts trivially on $T$, we have now shown that

$$
\frac{\left|L_{p}^{\left[a_{\text {inv }}(\mathcal{L})\right]}\right|}{\left|H^{0}\left(K_{p}, T\right)\right|}=\left|\operatorname{res}_{I_{p}}\left(L\left(T^{\prime}\right)_{p}^{\left[a_{\text {inv }}(\mathcal{L})\right]}\right)\right| .
$$

Preforming the same argument backwards for $T^{\prime}$ allows us to conclude

$$
\frac{\left|L_{p}^{\left[a_{\text {inv }}(\mathcal{L})\right]}\right|}{\left|H^{0}\left(K_{p}, T\right)\right|}=\frac{\left|L\left(T^{\prime}\right)_{p}^{\left[a_{\text {inv }}(\mathcal{L})\right]}\right|}{\left|H^{0}\left(K_{p}, T^{\prime}\right)\right|} .
$$

The $b$-invariant is the average value of these ratios for $p \leq x$ as $x \rightarrow \infty$ (see [1, Definition 4.6]) so that $b_{\text {inv }}(\mathcal{L})=b_{\text {inv }}\left(\mathcal{L}\left(T^{\prime}\right)\right)$. Note that for any nonzero $g \in\left(\left(i_{*}\right)^{-1} Y_{S}\right)^{\perp} \subseteq$ $H^{1}\left(K,\left(T^{\prime}\right)^{*}\right)$, we have $\operatorname{res}_{p}(g) \neq 0$ for a positive proportion of places $p$ by the Chebotarev density theorem. The coefficient of $p^{-a_{\text {inv }}\left(\mathcal{L}\left(T^{\prime}\right)\right) s}$ in $\widehat{i^{*} w}(g)$ is given by

$$
\sum_{f \in L_{p}^{\left[a_{\mathrm{inv}}(\mathcal{L})\right]}}\left\langle f, \operatorname{res}_{p}(g)\right\rangle p^{-a_{\mathrm{inv}}(\mathcal{L}) s},
$$

which is strictly smaller in absolute value than the coefficient of $p^{-a_{\text {inv }}\left(\mathcal{L}\left(T^{\prime}\right)\right) s}$ in $\widehat{i^{*} w}(0)$ for a positive proportion of $p$ by the fact that the Tate pairing is a perfect pairing. [1, Proposition 2.2] then shows that the singularity at $s=1 / a_{\text {inv }}\left(\mathcal{L}\left(T^{\prime}\right)\right)$ of $\widehat{i^{*} w}(g)$, if it is a singularity, is of strictly lower order than the rightmost pole of $\widehat{i^{*} w}(0)$. Thus, we know the rightmost pole for the generating function of $H_{\mathcal{L}\left(T^{\prime}\right)}^{1}\left(K, T^{\prime} ; X\right)$ does not cancel out and a Tauberian theorem implies that the order of magnitude of the main term is determined by the rightmost pole of $i^{*} w(0)$ so that

$$
\left|H_{\mathcal{L}\left(T^{\prime}\right)}^{1}\left(K, T^{\prime} ; X\right)\right| \sim c X^{1 / a_{\text {inv }}(\mathcal{L})}(\log X)^{b_{\text {inv }}(\mathcal{L})-1} .
$$


As this is a lower bound for $\left|H_{\mathcal{L}}^{1}(K, T ; X)\right|$ (up to the size of the finite kernel of $i_{*}$ ), we know the rightmost poles must not cancel out in this case either.

Corollary 1.2 is then a direct consequence of Theorem 1.1. We summarize the proof here, but we refer to [1] for the details.

Proof of Corollary 1.2 We remark that if $f \sim f^{\prime}$ under the coboundary relation, then there exists a $t \in T$ such that $(f * \pi)\left(G_{K}\right)=t(f * \pi)\left(G_{K}\right) t^{-1}$. Therefore, the $T$-conjugacy class of the image is preserved under the coboundary relation, so in particular surjectivity is preserved under the coboundary relation and the numerator in the corollary statement is well defined.

That the limit exists follows from the following inclusion-exclusion argument. For a subgroup $H \leq G$, let $\mathcal{L}(H \cap T)=\left(L_{p} \cap H^{1}\left(K_{p}, H \cap T\right)\right)_{p}$. (Here, and in what follows, we suppress pullbacks and pushforwards along the inclusion $H \cap T \hookrightarrow T$.) Then, as long as there exists at least one $f_{H} \in H_{\mathcal{L}}^{1}(K, T)$ such that $\left(f_{H} * \pi\right)(G) \leq H$, it follows that

$$
\left\{f \in H_{\mathcal{L}}^{1}(K, T ; X):(f * \pi)\left(G_{K}\right) \leq H\right\}=f_{H} * H_{\mathcal{L}(H \cap T), \operatorname{inv}_{f_{H}}}^{1}(K, H \cap T ; X)
$$

where $\operatorname{inv}_{f_{H}}(f)=\operatorname{inv}\left(f_{H} * f\right)$.

It is clear that $\mathcal{L}(H \cap T)$ and $\operatorname{inv}_{f_{H}}$ inherit the hypotheses of Theorem 1.1 from $\mathcal{L}$ and "inv" away from the places ramified in $f_{H}$, so Theorem 1.1 implies that

$$
\begin{aligned}
& \left|\left\{f \in H_{\mathcal{L}}^{1}(K, T ; X):(f * \pi)\left(G_{K}\right) \leq H\right\}\right| \\
& \sim c_{\mathrm{inv}_{f_{H}}}(K, \mathcal{L}(H \cap T)) X^{1 / a_{\mathrm{inv}_{f_{H}}}(\mathcal{L}(H \cap T))}(\log X)^{b_{\mathrm{inv}_{f_{H}}}(\mathcal{L}(H \cap T))-1} .
\end{aligned}
$$

Let $\mu_{G}$ be the Möbius function on the poset of $T$-conjugacy classes of subgroups $H \leq G$, ordered by inclusion up to conjugation, so that

$$
\begin{aligned}
& \frac{\mid\left\{f \in H_{\mathcal{L}}^{1}(K, T ; X): f * \pi \text { surjective }\right\} \mid}{\left|H_{\mathcal{L}}^{1}(K, T ; X)\right|} \\
& \sim \frac{\sum_{H} \mu_{G}(H) c_{\operatorname{inv}_{f_{H}}}(K, \mathcal{L}(H \cap T)) X^{1 / a_{\operatorname{inv}_{f_{H}}}(\mathcal{L}(H \cap T))}(\log X)^{b_{\operatorname{inv}_{f_{H}}}(\mathcal{L}(H \cap T))-1}}{c_{\text {inv }}(K, \mathcal{L}) X^{1 / a_{\text {inv }}(\mathcal{L})}(\log X)^{b_{\text {inv }}(\mathcal{L})-1}} .
\end{aligned}
$$

As the summation is a finite sum of functions of the form $c X^{a}(\log X)^{b-1}$, the limit certainly exists. (We know the limit is not infinite because the original ratio is bounded between 0 and 1.)

Part (c) of Corollary 1.2 then follows from a more in-depth study of the $a$ - and $b$ invariants to show that only the term $H=G$ of the summation contributes something nonzero to the limit, while parts (a) and (b) follow by bounding it below by a ratio of functions of the form $\left|H_{\mathcal{L}}^{1}(K, T ; X)\right|$ with the same $a$ - and $b$-invariants. The proof method for these steps is exactly the same as for [1, Theorem 5.3], so we direct the reader to that paper for the details.

In order to prove part (ii), it suffices to show that $\operatorname{disc}_{\pi}$ is admissible and Frobenian. This is proven in [1, Lemma 5.1]. 


\section{Non-periodic $w$}

In the following example, $w(f)$ will be a function of a complex variable $s \in \mathbb{C}$ so that

$$
\sum_{f \in H^{1}(K, T)} w(f)
$$

is a Dirichlet series, similar to the periodic example in Proposition 4.1. However, this examples will not be periodic and we will find that Theorem 2.3 decomposes this Dirichlet series as an infinite sum of Euler products.

We can still reference the Malle-Bhargava principle, which suggests that sum should have the same "order of magnitude" as $\hat{w}(0)$. Although this is not necessarily true for arbitrary infinite sums (even sums of Euler products), we are able to give evidence that this is close to the truth.

Proposition 5.1 Let $K$ be a number field, $S$ a finite set of places of $K$, and $T$ a Galois module. Define the function $w: H^{1}\left(\mathbb{A}_{K}, T\right) \rightarrow \mathbb{C}$ by

$$
w(f)= \begin{cases}\mathcal{N}_{K / \mathbb{Q}}(\operatorname{inv}(f))^{-s} & f \in \prod_{p} L_{p} \\ 0 & f \notin \prod_{p} L_{p},\end{cases}
$$

where

(a) $\mathcal{L}=\left(L_{p}\right)$ is a family of subsets $L_{p} \subset H^{1}\left(K_{p}, T\right)$ such that for all $p \notin S, H_{u r}^{1}\left(K_{p}, T\right) \subset$ $L_{p}$

(b) inv is an admissible ordering as defined in [1].

Then, w satisfies the hypotheses of Theorem 2.3.

Proof Simply note that $|w(f)|$ is bounded above by $\bar{w}(f)=\left|\mathcal{N}_{K / \mathbb{Q}}(\operatorname{inv}(f))\right|^{-s}$, the case that occurs when $L_{p}=H^{1}\left(K_{p}, T\right)$ is as large as possible. Note that $\bar{w}(f)$ is $Y_{S}$-periodic and satisfies the hypotheses of Theorem 2.3 by Proposition 4.1 . The remainder of the proof proceeds exactly like the proof of Proposition 4.1.

The difference between Propositions 5.1 and 4.1 is the removal of the translation invariance requirement on the family of allowable local conditions $\mathcal{L}$. The only condition we impose is that all but finitely many places allow for any unramified behavior, which we need to assume in order to avoid specifying the splitting types of infinitely many unramified primes. (If we did specify these, it might happen that no coclass satisfies all the local conditions!)

In this setting, Theorem 2.3 tells us that

$$
\sum_{f \in H^{1}(K, T)} w(f)=\frac{\left|H^{0}(K, T)\right|}{\left|H^{0}\left(K, T^{*}\right)\right|} \sum_{g \in H^{1}\left(K, T^{*}\right)} \hat{w}(g)
$$

is an infinite sum of Euler products. In order to apply a Tauberian theorem to get asymptotic information with these kinds of local restrictions, some more work needs to be done to uniformly control the order of $\hat{w}(g)$.

We present the simplest example of this phenomenon below:

Example 5.2 Let $K=\mathbb{Q}, T=C_{2}$ have the trivial action, and for each prime $p \nmid 2 \infty$, choose a subset $L_{p}$ with

$$
H_{u r}^{1}\left(\mathbb{Q}_{p}, C_{2}\right) \subsetneq L_{p} \subsetneq H^{1}\left(\mathbb{Q}_{p}, C_{2}\right) ;
$$


necessarily $\left|L_{p}\right|=3$. This amounts to choosing one of the two ramified quadratic extensions of $\mathbb{Q}_{p}$; the easiest choice, which we will make though it is not essential, is

$$
L_{p}=H_{u r}^{1}\left(\mathbb{Q}_{p}, C_{2}\right) \cup\left\{h_{p}\right\}, \quad h_{p} \text { corresponds to } \mathbb{Q}_{p}(\sqrt{p}) .
$$

Complete the family $\left(L_{p}\right)$ of local conditions by setting

$$
L_{2}=H_{u r}^{1}\left(\mathbb{Q}_{2}, C_{2}\right), \quad L_{\infty}=H^{1}\left(\mathbb{R}, C_{2}\right) .
$$

Then, counting cohomology classes satisfying the local conditions $L_{p}$, with respect to discriminant, amounts to counting squarefree integers $D \equiv 1 \bmod 4$ such that, for every $p \mid D, D / p$ is a square modulo $p$ : a cute question in arithmetic statistics, which we have not seen before.

If we define $w: H^{1}\left(\mathbb{Q}, C_{2}\right) \rightarrow \mathbb{C}$ as in Proposition 5.1 with $\mathcal{L}$ as above and the usual discriminant disc, then it follows (since $C_{2}$ is its own Tate dual) that

$$
\sum_{f \in H^{1}\left(\mathbb{Q}, C_{2}\right)} w(f)=\sum_{g \in H^{1}\left(\mathbb{Q}, C_{2}\right)} \hat{w}(g) .
$$

For an individual $g$, we see that

$$
\begin{aligned}
\hat{w}(g) & =\prod_{p \nmid 2 \infty}\left(\frac{1}{\left|H^{0}\left(\mathbb{Q}_{p}, C_{2}\right)\right|} \sum_{f_{p} \in L_{p}}\left\langle f_{p},\left.g\right|_{G_{\mathbb{Q}_{p}}}\right\rangle p^{-v_{p}\left(\operatorname{disc}\left(f_{p}\right)\right) s}\right) \\
& =\prod_{p \mid \operatorname{disc}(g)}\left(\frac{\left\langle h_{p}, g \mid \mathbb{Q}_{p}\right\rangle}{2} p^{-s}\right) \prod_{p \nmid 2 \operatorname{disc}(g) \infty}\left(1+\frac{\left\langle h_{p}, g \mid \mathbb{Q}_{p}\right\rangle}{2} p^{-s}\right) .
\end{aligned}
$$

The first product is of the form $\pm 2^{-\omega(\operatorname{disc}(g))}|\operatorname{disc}(g)|^{-s}$, while the second product is like an $L$-function. If $\chi_{g}$ is the character of $g$, then

$$
\prod_{p \nmid 2 \operatorname{disc}(g) \infty}\left(1+\frac{\left\langle h_{p}, g \mid \mathbb{Q}_{p}\right\rangle}{2} p^{-s}\right)=\sum_{2 \nmid n} \chi_{g}(n) 2^{-\omega(n)} \mu^{2}(n) n^{-s},
$$

which can be shown to be (conditionally) convergent and nonzero at $s=1$ unless $g=0$. If $g=0$, it has a pole of order $1 / 2$ at $s=1$. In order to conclude that $\hat{w}(0)$ really is the main term, we would need to show that (up to the signs of the summands)

$$
\sum_{g \in H^{1}\left(\mathbb{Q}, C_{2}\right)} 2^{-\omega(\operatorname{disc}(g))}|\operatorname{disc}(g)|^{-s} \sum_{2 \nmid n} \chi_{g}(n) 2^{-\omega(n)} \mu^{2}(n) n^{-s}
$$

is of the same or smaller "order of magnitude." This amounts to computing the moments of $L$-function look-alikes, and while in principle this is an accessible question, we will not address it in this paper.

\section{Author details}

${ }^{1}$ University of California San Diego, La Jolla, CA, USA, ${ }^{2}$ Princeton University, Princeton, NJ, USA.

Received: 7 May 2021 Accepted: 14 July 2021 Published online: 9 August 2021

\section{References}

1. Alberts, B.: Statistics of the first Galois cohomology group: a refinement of Malle's conjecture, December (2020). Preprint available at https://arxiv.org/abs/1907.06289, to appear in Algebra \& Number Theory

2. Bhargava, M.: Mass formulae for extensions of local fields, and conjectures on the density of number field discriminants. International Mathematics Research Notices, July (2010) 
3. Cohen, H., Lenstra, H.W.: Heuristics on class groups of number fields. Lect. Not. Math. Number Theory Noordwijkerhout 1983, 33-62 (1984)

4. Darmon, H., Diamond, F., Taylor, R.: Fermat's last theorem. Curr. Dev. Math. 1995(1), 1-154 (1995)

5. Frei, C., Loughran, D., Newton, R.: The Hasse norm principle for abelian extensions. Am. J. Math. 140(6), 1639-1685 (2018)

6. Frei, C., Loughran, D., Newton, R.: Number fields with prescribed norms October (2018). Preprint available at https:// arxiv.org/abs/1810.06024

7. Hewitt, E., Ross, K.A.: Abstract harmonic analysis. Vol. II: structure and analysis for compact groups. Analysis on locally compact Abelian groups. Die Grundlehren der mathematischen Wissenschaften, Band 152. Springer-Verlag, New York-Berlin (1970)

8. Jorza, A.: Math 160c Spring 2013 Caltech. Applications of global class field theory. Course notes, (2013). Available at www3.nd.edu/ ajorza/courses/m160c-s2013/overview/m160c-s2013.pdf

9. Neukirch, J., Schmidt, A., Winberg, K.: Cohomology of Number Fields, vol. 323, 2nd edn. Springer (2008)

10. O'Dorney, E.M.: Reflection theorems for number rings, July 2021. Preprint available at https://arxiv.org/abs/2107. 04727

11. Serre, J.-P.: Galois Cohomology. Springer-Verlag, Berlin (1997)

12. Tate, J.: Fourier analysis in number fields and Hecke's zeta-functions. In: Cassels, J.W.S., Frohlich, A. (eds.) Algebraic Number Theory. Thompson, Washington, DC (1950/1967)

13. Wright, D.J.: Distribution of discriminants of abelian extensions. Proc. Lond. Math. Soc. s3-58(1), 17-50 (1989)

\section{Publisher's Note}

Springer Nature remains neutral with regard to jurisdictional claims in published maps and institutional affiliations. 